\title{
New multiparameter bioanalytical technologies for applications in personalized medicine, drug discovery and fundamental biology
}

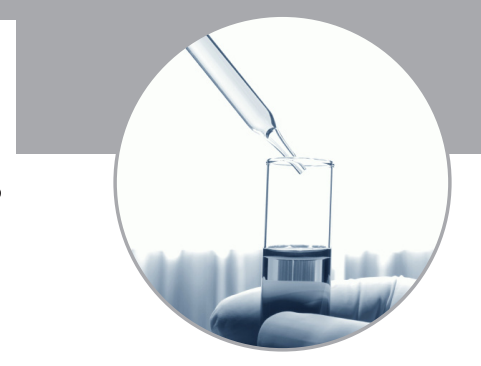

The ability to determine expression levels of multiple genetic and/or proteomic signatures simultaneously from the same sample volume is of tremendous importance in a wide range of biomedical applications, including disease diagnostics, drug discovery and fundamental studies of complex biological processes. Unfortunately, many of the tremendously enabling genomic and proteomic technologies available to the academic are not applicable in the clinical setting. Combining traditional chemical and biological insight with cutting-edge concepts from materials science, physics and engineering, our group brings an interdisciplinary approach to developing new tools in order to tackle longstanding refractory problems in the biological sciences - technologies that will enable fundamental biological discovery and, in some applications, expedite the transfer of biomolecular insight to the hands of the physician, where it can directly impact patient care.

\section{Why multiparameter analysis?}

This is an exciting time in biomedical science; with the sequencing of the human genome, researchers are armed with the fundamental blueprint of human life. Advances in genomic and proteomic technologies have continued to add more clarity to the complex picture of human biology and the perpetually growing body of available data, coupled with advances in computational approaches for data integration and correlation, have effectively ushered biomedical science into a golden age of information.

Global genomic and proteomic analyses have dramatically advanced our knowledge of the molecular basis of many human diseases. Our understanding of cancer biology, in particular, has greatly benefited from this 'information boom.' We now have an extensive list of causative genetic and epigenetic factors and purported proteomic disease biomarkers. However, equally, if not more, important is our improved understanding of biomolecular interconnectivity. Cascades of biomolecular interactions (or lack thereof) at the level of transcription and translation, in concert with post-transcriptional and post-translational events, propagate genetic perturbations that eventually result in diseased phenotypes. Referred to in different contexts as pathways, networks or systems, scientists are increasingly studying entire groups of interacting biomolecules to glean greater insight into the mechanism of disease onset and progression. The significance of this approach cannot be overstated since it highlights defects or mistakes within the biological system that represent 'weak links' or targets to which therapeutic agents can be effectively directed. Stated another way, multiparameter analysis not only answers the primary question 'is this cancer?', but can also provides insight toward answering the essential question 'how can I most effectively treat this disease?'

\section{Wanted: new multiparameter analysis technologies}

Our detailed understanding of the pathways, networks and/or systems that drive disease has been assembled using high-throughput genomic and proteomic technologies, in concert with immortalized cell lines and animal models. However, there can be significant differences between even the best models and 'real' human disease, on account of heterogeneity - both population heterogeneity and more subtle heterogeneities within patients themselves. To circumvent these complications, diagnoses must be performed at an individual level; individualized diagnosis may well be the most critical development needed to implement personalized approaches to medicine.

Unfortunately, many measurement approaches are not applicable in the clinic, where sample sizes and high technology-based instrumentation and expertise are limited. As a result, despite our increased knowledge of the molecular basis of human disease, the translation to clinical medicine has sadly lagged significantly behind. This is a measurement limitation, owing to the fact that current analytical techniques lack the compatibility, sensitivity and/or quantitative ability to simultaneously monitor expression levels of many genes and/or proteins from readily accessible body fluids or small solid tissue samples.

\section{Ryan C Bailey}

Department of Chemistry

\& Institute for Genomic Biology,

University of Illinois, Urbana-

Champaign, 600 S. Mathews

Avenue, MC 712, Box 45-5,

Urbana, IL 6180I, USA

Tel.: + I 2173330676

Fax: +I 2172656290

E-mail: baileyrc@illinois.edu
Multiparameter analysis

Simultaneous determination of the expression levels from multiple genomic, transcriptomic or proteomic signatures of disease 


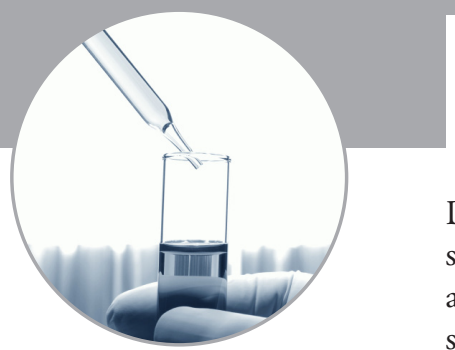

Developing new analytical tools to perform personalized multiparameter analysis on clinically accessible samples will greatly enhance patient survival and quality of life by reducing the need for invasive biopsy procedures and providing an informative diagnosis that will guide appropriate targeted molecular therapies to individuals.

A further consideration in the design of new multiparameter analysis technologies is the information that would ideally be obtained from a single patient sample. Traditional genomic, transcriptomic and proteomic technologies are inherently limited to independently analyzing DNA, RNA or proteins. However, it is becoming increasingly clear that information flow between levels (Figure I), and the correlations or discordance in expression, contain an incredible amount of disease-specific information. Attempts to understand information flow are currently hindered by the requirements for multiple analysis methodologies and sample volumes. This, in turn, leads to difficulties in data correlation and interpretation, in light of differences in sample preparation, tissue heterogeneity and cross-platform data correlation. Therefore, a 'wish list' for new technology development might include the ability to monitor, on a single analytical platform, the expression of DNA, RNA, miRNA, proteins and post-translationally modified proteins [1].

\section{New multiparameter analysis technologies in development by the Bailey group}

Motivated by practical challenges associated with performing multiparameter biological analysis, our group is developing a suite of powerful

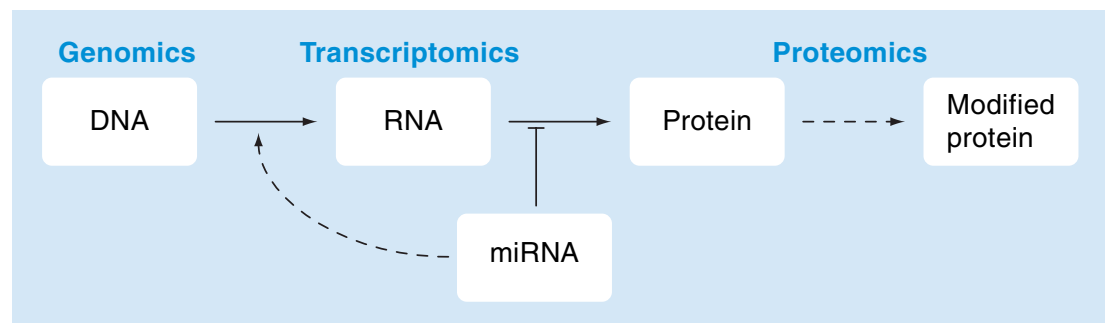

Figure 1. Biological information flows from the level of DNA to RNA and on to proteins. Post-transcriptional effects from miRNAs and post-translational modifications of proteins add additional layers of biological complexity. Information about disease onset and progression can be extracted from conventional measurements of DNA, RNA or proteins; however, valuable insight can also be extracted from analyzing the correlations or discordance as information flows between levels. New analytical methods that can simultaneously perform measurements across multiple levels of biomolecular complexity will be extraordinarily valuable in understanding the propagation of perturbations that result in diseased phenotypes. analysis tools that will be applicable in clinical settings and beyond. Our goal is to facilitate personalized diagnosis and individualized treatment by providing a more detailed picture of the biomolecular signatures of disease from a single patient. Due to their simplicity, scalability and molecular generality, these tools also have broad applicability to many aspects of pharmaceutical research and fundamental biological studies.

\section{Silicon photonic microring resonators}

We are developing an incredibly enabling biological analysis technology based upon high-density arrays of silicon photonic microring resonators [2]. The basis of operation for this technology is quite simple, as illustrated in Figure 2. Optical modes supported within microcavities are very sensitive to the local refractive index near the microring surface. When molecules of DNA, RNA or protein bind to an appropriate capture probe, immobilized on the microring surface, the refractive index changes and the frequencies of optical resonances shift in a well-defined manner. It is important to note that this is a label-free detection technology and, therefore, no fluorescent, enzymatic or radioactive probes are required. From a practical perspective, labelfree methods are often advantageous in terms of reducing assay cost and complexity and also avoiding deleterious effects that the signaling moiety itself might have on the biomolecular interaction being studied [3].

Many thousands of microring resonators can be fabricated with tremendous precision on the wafer scale, using commercially validated semiconductor processing techniques. Furthermore, each microring sensor can be uniquely encoded with a different capture agent (e.g., cDNA or antibodies) using conventional microarraying tools, and operation of these devices in a frequency region used by existing optical telecommunications technologies allows us to construct a robust and easy-to-use sensing methodology with on-chip integrated optical components, microfluidic fluid actuation and biofouling resistant coating strategies. The ability to apply these existing technologies to an entirely different application leads toward an extremely sensitive, highly multiplexable detection platform, which should be applicable to many clinical and pharmaceutical discovery applications.

Although it is still in the early stages of development, we have demonstrated the applicability of the technology to the detection of DNA, miRNAs and protein biomarkers using 
silicon photonics chips similar to that shown in Figure 3. This version of the sensor chip has 64 uniquely addressable sensors that can be independently interrogated. The presence of many sensors on the same chip allows different modes of operation - many unique assays can be run in parallel or a smaller number of assays can be performed multiple times in parallel. The ability to perform many identical measurements in the same small $(<100 \mu \mathrm{l})$ sample volume increases measurement precision and reduces the number of false-positive responses. Furthermore, additional microrings in the assay chamber that are not functionalized to be selective for a particular target analyte can greatly alleviate matrix effects by serving as control sensors to correct for biofouling, small temperature variations and other sources of nonspecific response.

As an initial demonstration of the technology for proteomic applications, we have performed label-free quantitation of the cancer biomarker carcinoembryonic antigen (CEA) with a limit of detection comparable to, and precision exceeding that of, commercial ELISA assays [4]. We have also shown that assay dynamic ranges can exceed four orders of magnitude and that measurements can be made in media as complex as undiluted blood serum with only a small reduction in absolute sensitivity. We have applied this technology to the sensitive quantitation of cytokines and have also shown that label-free multiplexed assays, utilizing six different cancer biomarker-specific capture agents arrayed in triplicate, can be performed with absolutely no loss in sensitivity - each reaching clinically relevant levels. Another important proteomic application of the technology lies in the multiplexed screening of protein-protein interactions. The ability to simultaneously determine the kinetic association, dissociation and equilibrium constants of multiple protein-protein interactions has applications throughout drug target determination, antibody screening and fundamental biology.

We have also shown that microring resonators can be effectively utilized to detect nucleic acids with high sensitivity [5]. In particular, we are developing a hybridization-based multiplexed assay for profiling the expression levels of multiple miRNAs, without additional steps of ligation, amplification or labeling. Preliminary experiments show that the platform is significantly more sensitive than traditional Northern blotting methods of detection and that sample size requirements are smaller than many commercial miRNA analysis kits. In addition, we have begun to utilize our microring resonator platform to investigate protein-nucleic acid and protein-carbohydrate binding interactions.

Based on the platform's sensitivity and scalability, as well as its ability to detect both proteins and nucleic acids, we believe label-free microring resonator sensors are a promising tool for multiparameter analysis in clinical, pharmaceutical and fundamental biological applications. Much work remains to be performed, however this technology development effort is, in part, aimed at translating biological insight into improvements in clinical medicine, by empowering physicians with the same biomolecular information available to academics.

\section{- Multicomponent strategies for assessing tissue heterogeneity}

One challenge underlying many aspects of tissue-based disease diagnosis is sample heterogeneity. It is well established that cell-to-cell heterogeneity is present within even the simplest biological systems and is a critical determinant in processes such as embryonic development and disease onset and progression. Therefore, the critical biomolecular signatures of disease can be best elucidated from homogeneous samples. One of the most pronounced examples of dynamic tissue heterogeneity lies in the metastasis of cancerous tumors. Metastasis, as with normal cellular adhesion, is controlled by interactions with many hundreds of different biomolecules, including proteins, carbohydrates and lipids. Substrates that present only a single

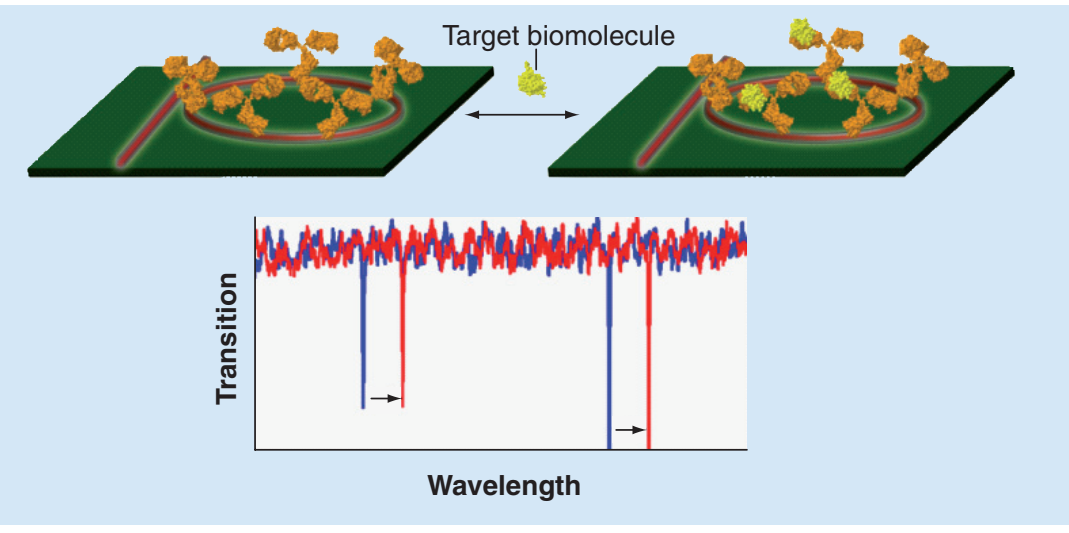

Figure 2. Microring resonator biosensor. The microring on the left is functionalized with a capture agent (an antibody is shown here) and has an optical resonance spectrum shown in blue. Binding of the target biomolecule changes the local refractive index surrounding the microring on the right, which in turn causes a shift in the optical resonances (red trace). By monitoring the frequency shift of resonant peaks accompanying sample introduction, the concentration of biomolecular species can be quantitatively determined. 


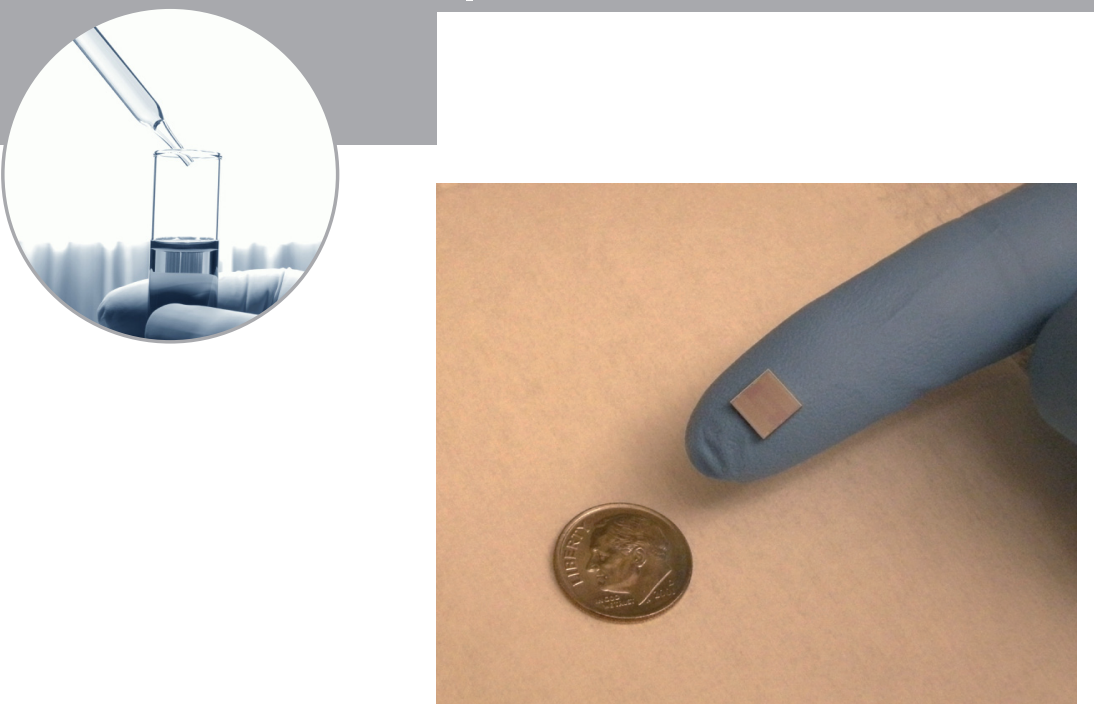

Figure 3. Silicon photonics chip containing 64 microring optical resonators, which can be uniquely functionalized with multiple capture agents against different biomolecular targets. Each microring sensor can be individually addressed to determine the concentration of different biomolecules from within a single sample. Optimization of device geometry should enable sensor densities in excess of 10,000 per $\mathrm{cm}^{2}$.

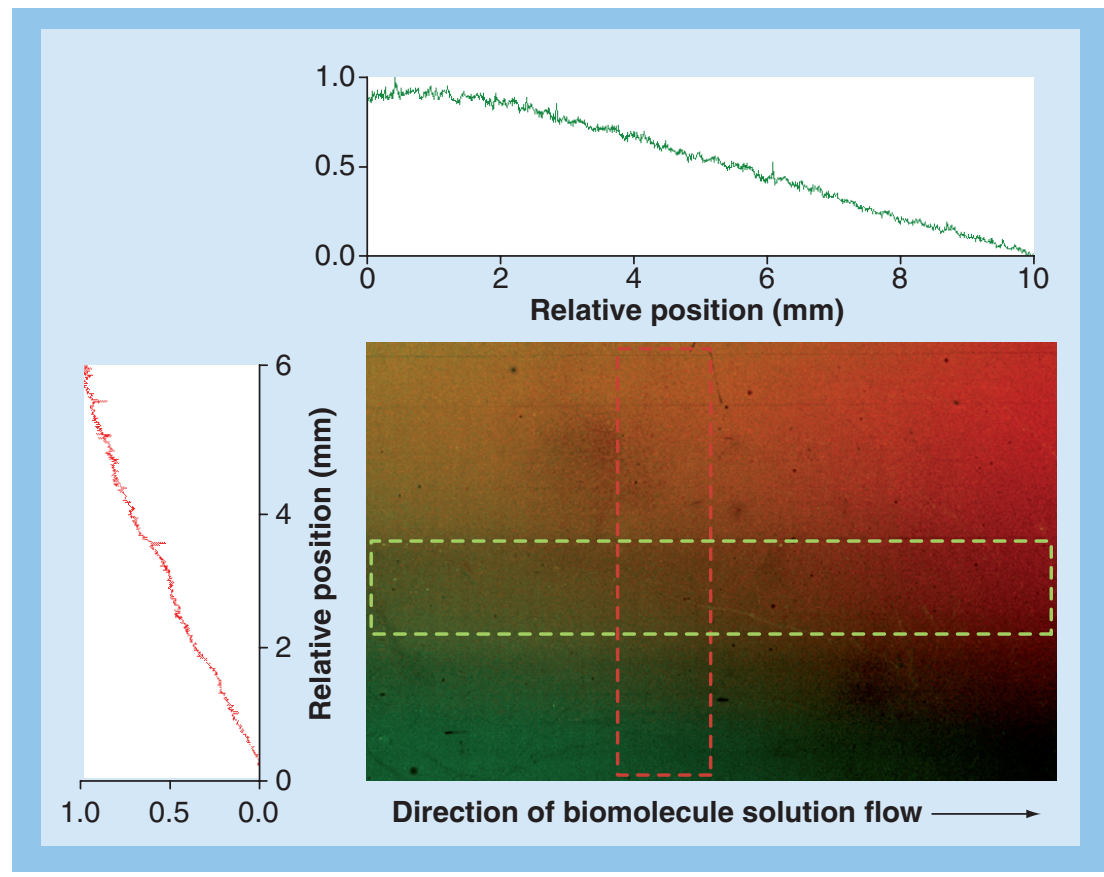

Figure 4. Fluorescence scan of a $10 \times 6-\mathrm{mm}$, two-component photopatterned gradient surface and corresponding line traces to illustrate the gradient geometry. From left to right, the concentration of mannan (green, visualized by secondary staining with a lectin) decreases in a controllable manner. Similarly, from top to bottom, the concentration of P-selectin (red, visualized by secondary staining with an antibody) is systematically controlled. The simultaneous control over the density of both immobilized biomolecules is significant as a large number of concentration combinations are represented on one substrate and, thus, can be interrogated in a single experiment, from a single sample.

Reproduced with permission from the American Chemical Society [6]. biomolecule can be very valuable in understanding disease-altered adhesion; however, we believe that a more complete picture will be obtained by developing more complex models that include multicomponent biomolecular contributions.

In order to better understand the molecular determinants that dictate normal and diseaseinduced perturbations in cell adhesion, we are developing methods of controlling the spatial presentation and density of multiple biomolecules on model substrates. Our method, based upon photochemical attachment, is molecularly universal and can create surfaces with overlapping patterns and gradients of multiple biomolecules, as shown in Figure 4 [6]. Importantly, gradient structures present continually varying compositions of multiple different receptors. We envision several applications of the resulting substrates, including:

- A heterogeneous sample might stratify according to small differences in phenotype, due to different cell-surface interactions at different spatial locations;

- The effects of competing surface interactions on a clonal population of cells could be observed in a single experiment by culturing the population on a multicomponent gradient surface.

\section{Outlook for the future}

The future of biomedicine lies in the ability to measure and integrate the expression levels of multiple biomolecular signatures in order to better understand normal human biology, as well as disease onset and progression. The powerful genomic and proteomic technologies that have provided the motivation for these studies are ill equipped, at present, to take the next step - particularly in clinical settings. It is likely that new methodologies will need to be developed to meet these emerging measurement challenges. Our group is developing two such multiparameter analysis technologies, which we hope will develop into valuable tools to provide physicians with personalized disease fingerprints. Appropriate multiparameter analysis technologies will also contribute to our understanding of how pharmaceuticals can be developed and prescribed most effectively. In the future, we envision that personalized diagnostics will transform cancer classification from organ of origin to a characteristic set of specific biomolecular perturbations that are known to respond to a particular treatment regimen for that individual. The development of multiparameter assays will likely 
become increasingly coupled to pharmaceutical development, leading to more effective clinical trials conducted on the most appropriate subset of patients and, ultimately, improving patient survival and quality of life.

\section{Financial \& competing interests disclosure}

The author acknowledges financial support for the group's efforts in developing new multiparameter biomolecular analysis methods from the following agencies: the NIH Director's New Innovator Award Program, part of the NIH Roadmap for Medical Research, through grant number 1-DP2-OD002190-01; the Roy J Carver Charitable Trust; a New Faculty Grant from the Camille and Henry Dreyfus Foundation and a 3M Non-Tenured Faculty Grant. The author has no other relevant affiliations or financial involvement with any organization or entity with a financial interest in or financial conflict with the subject matter or materials discussed in the manuscript. This includes employment, consultancies, honoraria, stock ownership or options, expert testimony, grants or patents received or pending, or royalties.

No writing assistance was utilized in the production of this manuscript.

\section{Executive summary}

- Multiparameter biomolecular analysis can generate a detailed picture of the combined genetic and proteomic perturbations that underlie disease onset, provide information on patient prognosis and illuminate potential therapeutic strategies.

- Presently available analytical tools are limited in their multiplexing capabilities, particularly in the simultaneous analysis of DNA, RNA and proteins, and their applicability to a clinical setting where sample sizes are limited and assay time-to-result is of tremendous importance. Exciting opportunities exist for the development of new diagnostic technologies.

- Silicon photonic microring resonators represent a promising platform for highly multiplexed, label-free bioanalysis due to their sensitivity, commercially-validated fabrication and applicability to the direct measurement of many genes and proteins from a single sample volume.

- The understanding of normal and disease-altered cell adhesion will be further increased using multicomponent model substrates that display multiple different biomolecules at controlled surface densities and in overlapping geometric arrangements.

\section{Biography}

Ryan C Bailey is an Assistant Professor in the Department of Chemistry and the Institute for Genomic Biology and an affiliate of the Micro and Nanotechnology Laboratory at the University of Illinois at Urbana-Champaign. He received his BSc from Eastern Illinois University and his PhD in Chemistry from Northwestern University, where he worked with Joseph Hupp to create a new chemical and biological sensing methodology based upon optical diffraction gratings. He then worked jointly as a postdoctoral fellow with James Heath at the California Institute of Technology and with Leroy Hood at the Institute for Systems Biology, and developed the DNA-encoded antibody library strategy for multiplexed immunoassays, the core technology behind the integrated blood-barcode chip. Research in Ryan Bailey's laboratory in Illinois is broadly focused on developing new tools to enable multiparameter analysis of complex biological samples with applications in individualized disease diagnostics and theragnostics, drug discovery and fundamental biology.

\section{Bibliography}

1 Bailey RC, Kwong G, Radu CG, Witte ON, Heath JR. DNA-encoded antibody libraries: a unified platform for multiplexed cell sorting and detection of genes and proteins. J. Am. Chem. Soc. 129, 1959-1967 (2007).

2 Bailey RC, Washburn AL, Qavi AJ et al. A robust silicon photonic platform for multiparameter biological analysis. Proc. SPIE $7220,72200 \mathrm{~N}$, DOI:10.1117/12.809819 (2009).
3 Qavi AJ, Washburn AL, Byeon J-Y, Bailey RC. Label-free technologies for quantitative multiparameter biological analysis. Anal. Bioanal. Chem. 394, 121-135 (2009).

4 Washburn AL, Gunn LC, Bailey RC. Label-free quantitation of a cancer biomarker in complex media using silicon photonic microring resonators. Submitted (2009).
5 Qavi AJ, Bailey RC. Multiplexed detection and label-free quantitation of microRNAs using arrays of silicon photonic microring resonators. Submitted (2009).

6 Toh CR, Fraterman TA, Walker DA, Bailey RC. Direct biophotolithographic method for generating substrates with multiple overlapping biomolecular patterns and gradients. Langmuir 25, 8894-8898 (2009). 